\title{
Toda lesão do diafragma por ferimento penetrante deve ser suturada?
}

\section{Must all diaphragmatic penetrating wounds be sutured?}

\author{
Roberto SAAd Júnior, TCBC- SP'; Roberto Gonçalves, TCBC-SP²
}

\author{
R E S U M O
}

\begin{abstract}
Os ferimentos da região tóracoabdominal podem cursar com ferimentos diafragmáticos em até $48 \%$ dos casos. Lesões associadas ao trauma diafragmático, estão presentes na maioria das vezes, o que facilita o diagnóstico da lesão diafragmática e em consequência impõe o tratamento operatório. Porém em 8 a 10\% dos casos, as lesões diafragmáticas são isoladas e por apresentarem pouco ou nenhum sintoma, estas lesões podem passar despercebidas. Nestas situações, o diafragma poderia ser tratado de modo conservador, sem a sutura do mesmo? Após a realização de trabalhos experimentais com animais e de analisar a literatura, concluímos que ainda não podemos afirmar com certeza de que é possível não suturar um ferimento diafragmático.
\end{abstract}

Descritores: Ferimentos penetrantes. Diafragma. Traumatismos abdominais. Traumatismos torácicos. Suturas.

\section{INTRODUÇÃO}

A tendência atual para o tratamento de lesões intraabdominais advindas de traumatismos fechados ou penetrantes, desde que possível e seguindo todos os preceitos já conhecidos, é o de evitar o tratamento operatório. Esta conduta tem se revelado verdadeira para casos selecionados, de modo que, para estes doentes o tratamento não operatório é a solução ideal, evitando assim uma laparotomia que sem dúvida expõe o doente a mais um trauma e à complicações inerentes à operação.

Seguindo esta tendência, o tratamento não operatório, das lesões diafragmáticas seria viável?

Para responder esta pergunta, temos que entender qual a história natural de um ferimento diafragmático e ainda especificar melhor, onde esta situação mereceria ser analisada. É o que tentaremos discutir neste capítulo.

\section{Ferimentos penetrantes e lesões diafragmáticas}

A principal causa de lesão diafragmática é causada por ferimentos penetrantes na zona de transição tóraco-abdominal ${ }^{1}$, delimitada anteriormente pelo quarto espaço intercostal, sexto espaço intercostal lateralmente, ponta da escápula posteriormente e como limites inferiores a região epigástrica e rebordos costais ${ }^{2}$. Os ferimentos desta zona podem cursar com lesão diafragmática em 20 a $40 \%$ dos $\operatorname{casos}^{3}$ ou até $48 \%{ }^{4}$.No entanto a real incidência é desconhecida pelo grande número de doentes com lesões assintomáticas inicialmente após o trauma, principalmente nas causadas por arma branca, sendo que o diagnóstico só será realizado, neste casos, tardiamente quando os sintomas aparecerem ou nas complicações.

Lesões associadas ao trauma diafragmático estão presentes na maioria das vezes sendo o pulmão, estômago, fígado, baço e colón os órgãos mais lesados porém em 8 a 10\% as lesões diafragmáticas são isoladas ${ }^{5}$ evoluindo com poucos ou nenhum sintoma e por isto mesmo de difícil diagnóstico.

Por razões já descritas anteriormente, uma lesão diafragmática na fase aguda do trauma pode passar despercebida. O exame físico, a radiografia de tórax, ultrasonografia, tomografia computadorizada e o lavado peritoneal diagnóstico, juntos não tem sensibilidade nem especificidade boas para o diagnóstico das lesões diafragmáticas com índices de 10 a 30\% de lesões não diagnosticadas, principalmente quando são isoladas ${ }^{6}$. Sendo assim em alguns Serviços ${ }^{7}$ adotou-se a Videocirurgia como meio de se diagnosticar estas lesões diafragmáticas oligo ou assintomáticas que de outro modo passariam sem o diagnóstico. Doentes que chegam com ferimentos da zona de transição tóraco-abdominal, suspeitos portanto de possuírem uma lesão do diafragma e que não apresentam sintomas ou que os exames de imagem ou laboratoriais

1. Professor Titular da Disciplina de Cirurgia Torácica do Departamento de Cirurgia da Faculdade de Ciências Médicas da Santa Casa de São Paulo FCMSCSP-SP-BR; 2. Professor Instrutor do Departamento de Cirurgia da Faculdade de Ciências Médicas da Santa Casa de São Paulo-SPBR. 
convencionais não revelaram qualquer alteração do músculo diafragmático, mesmo assim são submetidos à videocirurgia, para só desta maneira trazer a certeza da existência ou não desta lesão.

Uma vez feito o diagnóstico, e desde a clássica descrição de Carter e Giuseffï, que definiram que por esta lesão diafragmática órgãos abdominais atravessariam este orifício com formações das hérnias e que os doentes portadores desta afecção apresentariam três fases de evolução: fase aguda, fase de latência e a fase tardia com altas taxas de morbilidade e mortalidade, justifica-se o tratamento operatório para suturar o diafragma e prevenir tais complicações.

\section{Ferimentos Penetrantes - Fase Aguda}

Estas lesões podem ser classificadas da seguinte maneira: lesão da zona de transição toracoabdominal com sinais de peritonite; lesões da zona de transição torácoabominal com sinais de comprometimento de órgãos torácicos que necessitam de toracotomias para as suas correções; lesões da zona de transição tóracoabdominal seguidas de imediata herniação visceral abdominal para o tórax; lesões da zona de transição tóraco-abdominal, hemodinamicamente instáveis. Nestas situações é evidente que o tratamento cirúrgico é necessário, pois além de corrigir as lesões existentes há necessidade de tratar a lesão do diafragma, se presente. Não há o que se discutir; esta é a melhor conduta ${ }^{9}$, aqui não há lugar para o tratamento conservador.

Estas lesões da zona de transição toracoabdominal também podem ocorrer com: lesão isolada do diafragma diagnosticada; lesão isolada do diafragma despercebida; lesão do diafragma associada a um tipo de lesão de víscera abdominal que permite um tratamento não operatório. Nestas três situações acreditamos que podemos e devemos analisar, a possibilidade de não suturar o diafragma. Não conhecemos a história natural das lesões diafragmáticas, mas sabemos que algumas evoluem com hérnia diafragmática.

Fica a pergunta: todas as lesões diafragmáticas evoluem para a herniação ou existe a possibilidade de cicatrização e cura sem necessariamente a formação de uma hérnia?

Ferimentos Penetrantes - Fase de Latência

Após meses ou anos do trauma, o doente pode apresentar-se com sintomas digestivos ou respiratórios devido à presença de uma hérnia diafragmática. Esta lesão que não existia na fase aguda do trauma formou-se lenta e progressivamente em decorrência da gradual passagem de víscera abdominal para a cavidade pleural. Esta herniação resulta principalmente do gradiente de pressão pleuroperitoneal entre $7 \mathrm{~cm}$ e $20 \mathrm{~cm} \mathrm{H}_{2} \mathrm{O}$ durante a respiração normal e de até $100 \mathrm{~cm} \mathrm{H} \mathrm{H}_{2}$ na inspiração profunda. Também nesta situação a operação é mandatória, não há que se discutir.

\section{Ferimentos Penetrantes - Fase Tardia}

A fase tardia é representada pelas complicações decorrentes da hérnia. O encarceramento da víscera herniada determina quadro obstrutivo que em até $20 \%$ dos casos pode evoluir para necrose da víscera caracterizando o seu estrangulamento. Nesta fase, a mortalidade relatada é de $16 \%$ a $20 \%$ para os casos de obstrução e de até $80 \%$ para o estrangulamento ${ }^{2}$. Novamente nesta situação não há como, não se indicar a operação, inclusive com a sutura diafragmática.

\section{História natural das lesões diafragmáticas Estudo experimental}

Com a intenção de se obter uma história natural do ferimento diafragmático e verificar se ocorre ou não a cicatrização espontânea deste músculo após um ferimento penetrante realizamos alguns estudos que começaram esclarecer este fenômeno.

Perlingeiro et al. ${ }^{10}$,em 2001, utilizando modelo murino, provocaram a lesão experimental de $10 \%$ da superfície do diafragma através de videolaparoscopia, sob anestesia geral. Os animais foram distribuídos em dois grupos quanto ao acompanhamento temporal (precoce e tardio), sendo que os do grupo precoce foram mortos entre 11 e 60 dias de pós operatório e os animais do grupo tardio foram mortos entre 120 a 150 dias. Após a morte dos animais foram analisados aspectos macroscópicos com presença ou ausência de hérnia diafragmática, cicatrização espontânea e estudo microscópico. Não houve significância estatística em relação ao tempo de evolução, apresentando cicatrização espontânea em 91,1\% do total ( $n=56$ ratos). As lesões foram realizadas tanto no diafragma direito como no diafragma esquerdo. Houve cicatrização em $100 \%$ das lesões à direita e $83 \%$ à esquerda.

O interessante foi que em 33,9\% não foi possível identificar o local da cicatrização, sugerindo ter ocorrido regeneração muscular ${ }^{10}$. As alterações histológicas encontradas nos diafragmas cicatrizados foram: ausência de lesão, infiltrado inflamatório e/ou alterações reparadoras, comprometimento neurogênico exclusivo e alterações inflamatórias e ou reparadoras em associação ao comprometimento neurogênico. Este foi o primeiro trabalho de uma série que está mostrando a capacidade de cicatrização espontânea do músculo diafragmático.

Gonçalves em $2008^{11}$, repetiu o trabalho anterior também com murinos, no entanto, provocou lesões maiores, equivalentes a $30 \%$ de extensão e somente no diafragma esquerdo. Neste estudo não foi avaliado o diafragma direito. Após 7, 14 e 21 dias os animais foram mortos. Observou-se ao final do experimento a evolução da lesão provocada: cicatrização espontânea e presença ou ausência de hérnia diafragmática. Encontrou: em 65\% presença de hérnia diafragmática, em 22,5\% lesão diafragmática sem herniação e em 12,5\% cicatrização 
espontânea. As análises histológicas dos espécimes que apresentaram cicatrizações espontâneas mostraram invariavelmente infiltrado monocelular, proliferação de fibroblastos, neoformação vascular e deposição de colágeno. Os espécimes que apresentaram lesões sem herniação dos órgãos abdominais para o tórax assim como o grupo que apresentou hérnia diafragmática, mostraram fibrose associada ao infiltrado inflamatório crônico. Estes achados evidenciam que as lesões maiores do diafragma esquerdo, que não possuem a suposta proteção do fígado, também têm capacidade de cicatrização, embora em número inferior ocorrido no trabalho anterior.

Rivaben em 200912, analisou a evolução natural de ferimentos penetrantes, com extensão de $30 \%$, no diafragma, no lado direito, durante 21 dias em 28 ratos e verificou que ocorrera cicatrização espontânea em 54\% das lesões.

Estes estudos ${ }^{10-12}$ sugerem que: ferimentos pequenos no diafragma, a maioria cicatriza, tanto do lado direito como do lado esquerdo; ferimentos maiores também podem cicatrizar, no entanto, aqueles do lado direito do diafragma são os que têm maior chance de cicatrização. Houve cicatrização dos ferimentos do lado esquerdo diafragma, porém em um pequeno número de casos, mostrando aí, talvez o poder protetor do fígado na prevenção de uma hérnia diafragmática; o tempo necessário para possíveis cicatrizações é de 21 dias.

\section{DISCUSSÃO}

Em 1994, Renz et al. ${ }^{13}$ publicaram o resultado do tratamento não operatório de doentes portadores de ferimentos tóracoabdominais do lado direito. Foram analisados 13 doentes hemodinamicamente estáveis e a drenagem pleural foi realizada em todos. Nenhum deles, durante a admissão, apresentava sinais de peritonite. Lesões de pulmão ou do fígado estavam presentes. Período do estudo: entre 1990 a 1994. Estes autores acreditam que doentes selecionados com este tipo de ferimento poderão ser tratados de modo não operatório, pois no período de acompanhamento, não verificaram maiores complicações. Neste estudo nada foi comentado sobre eventual ferimento diafragmático, se presente ou não e qual o melhor método de tratamento caso estas lesões estivessem presentes.

Acreditamos que estes doentes apresentavam também lesões diafragmáticas; pois bem, o que aconteceu com elas: cicatrizaram? Não cicatrizaram e daí a possibilidade tardia de aparecimentos de hérnias com todas suas complicações? Estas respostas não tivemos e, portanto, não podemos ter a certeza de que o tratamento não operatório nestes doentes foi a melhor conduta.

Sempre foi o maior temor do cirurgião deixar de fazer diagnóstico de lesões diafragmáticas nestes tipos de ferimentos ${ }^{6,14}$. A literatura apresenta séries mostrando a alta incidência de lesões diafragmáticas nestas circunstâncias $^{2,15}$.

Outro aspecto importante é o fato de que mesmo que a lesão passe despercebida na fase aguda do trauma e o doente tenha alta hospitalar, não significa que está curado, pois, complicações tardias aparecem com freqüência razoável, mesmo anos após o acidente ${ }^{16,17}$. De tal modo, que, já apreendemos que não é seguro tratar de modo conservador uma lesão diafragmática, pois todos fomos testemunhas, de verdadeiras catástrofes em consequência de complicações tardias, quando a lesão diafragmática não foi diagnosticada e adequadamente tratada.

\section{ANÁLISE CRÍTICA}

Toda lesão do diafragma por ferimento penetrante deve ser suturada? No atual estágio do conhecimento fica muito difícil responder a esta pergunta. Existem argumentos contrários e a favor desta conduta. Um argumento favorável ao tratamento cirúrgico das lesões diafragmáticas advoga que se elas não forem suturadas na fase aguda poderão advir complicações, meses ou anos após o trauma, com altas taxas de morbidade e de mortalidade.

Aqueles que defendem o tratamento conservador argumentam que: pelo diagnóstico cada vez mais freqüente de lesões diafragmáticas por trauma penetrante, principalmente quando estes ocorrem na transição tóracoabdominal, deve haver algum tipo de cicatrização espontânea em parte destes ferimentos, caso contrário teríamos um número maior de hérnias, pois o número de lesões potenciais que não são operados é muito maior que o de hérnias 6,18; os trabalhos experimentais realizados em ratos $^{10-12}$, que mostraram a possibilidade de cicatrização espontânea do diafragma, o que não é definitivo, posto que, foram experimentos em animais, no entanto, já é um começo.

No estágio atual do conhecimento não é recomendável que se realize o tratamento não operatório de lesões diafragmáticas, mas em um futuro bem próximo com advento de novas tecnologias principalmente no campo de imagem (tomografia de corpo inteiro, reconstrução de imagens e tomografias) faremos o diagnóstico destas lesões com mais facilidade e mais precisão e a partir daí com o diagnóstico realizado, poderemos optar por um tratamento conservador ou operatório. Pelos trabalhos experimentais realizados, talvez os ferimentos diafragmáticos pequenos e os do lado direito são aqueles, com mais possibilidades de cicatrização espontânea e portanto admitirão um tratamento conservador.

\section{CONSIDERAÇÕES FINAIS}

Uma lesão diafragmática pequena e à direita poderá cicatrizar espontaneamente e ser tratada de modo 
conservador desde que: tenhamos o diagnóstico preciso desta lesão na fase aguda do trauma; seja feito o acompanhamento do doente por longo período de tempo pois, caso a lesão não cicatrize, teremos como conseqüências graves complicações com altos índices de morbidade e de mortalidade. Não há estudos na literatura nacional ou internacional que mencione séries de doentes que foram assim tratados e acompanhados por longo período de tempo. De modo que as afirmações acima são afirmações teóricas e este campo permanece aberto.

\section{A $B$ S T T R A C T}

Wounds to the abdominal-thoracic region are associated with diaphragm wounds in up to $48 \%$ of cases. Lesions secondary to diaphragm trauma are present in the majority of cases, facilitating the diagnosis of diaphragm lesion and subsequent surgical management. However, diaphragm lesions are isolated in 8 to 10\% of cases and because they present few or no symptoms may be overlooked. In such situations, can the diaphragm be treated conservatively without suturing? Based on experiments in animals and a review of the literature, we concluded there is currently insufficient evidence to affirm that diaphragm injuries require no suturing

Key words: Wounds, penetrating. Diaphragm. Abdominal injuries. Thoracic injuries. Sutures.

\section{REFERENCIAS}

1. Asencio JA, Demetriades D, Rodrigues A. Injury to the diaphragm In: Moore EE, Mattox KL, Feliciano DV, editores. Trauma. 4th New York: McGraw-Hill; 2000. p. 603-32.

2. Madden MR, Paull DE, Finkelstein JL, Goodwin CW, Marzulli V Yurt RW, et al. Occult diaphragmatic injury from stab wounds to the lower chest and abdomen. J Trauma. 1989;29(3):292-8.

3. Zantut LF, Ivatury RR, Smith RS, Kawahara NT, Porter JM, Fry WR, et al. Diagnostic and therapeutic laparoscopy for penetrating abdominal trauma: a multicenter experience. J Trauma. 1997;42(5):825-9; discussion 829-31.

4. Ivatury RR, Simon RJ, Stahl WM. A critical evaluation of laparoscopy in penetrating abdominal trauma. J Trauma. 1993;34(6):822-7; discussion 827-8

5. Gianini JA. Ferimentos penetrantes tóraco-abdominais e de tórax e abdome. Análise da morbilidade e mortalidade pós-operatória [dissertação]. São Paulo: Santa Casa de São Paulo, Faculdade de Ciências Médicas; 1996.

6. Demetriades D, Kakoyiannis S, Parekh D, Hatzitheofilou C. Penetrating injuries of diaphragm. Br J Surg. 1988;75(8):824-6.

7. Saad Jr R, Carvalho WR, Ximenes Netto M, Forte V. Cirurgia torácica geral. São Paulo: Atheneu; 2005.

8. Carter BN, Giuseffi J. Strangulated diaphragmatic hernia. Ann Surg. 1948;128(2):210-25.

9. Arak T, Solheim K, Pillgram-Larsen J. Diaphragmatic injuries. Injury. 1997;28(2):113-7.

10. Perlingeiro JA, Saad R Jr, Lancelotti CL, Rasslam S, Candelária PC Soldá SC. Natural course of penetrating diaphragmatic injury: an experimental study in rats. Int Surg. 2007;92(1):1-9.

11. Gonçalves R. Análise da evolução natural das feridas pérfurocortantes equivalentes a $30 \%$ do diafragma esquerdo. Estudo experimental em ratos [dissertação]. São Paulo: Santa Casa de São Paulo, Faculdade de Ciências Médicas; 2008.
12. Rivaben JH. História natural do ferimento diafragmático extenso à direita: estudo experimental em ratos [dissertação]. São Paulo: Santa Casa de São Paulo. Faculdade de Ciências Médicas; 2011

13. Renz BM, Feliciano DV. Gunshot wounds to the right thoracoabdomen: a prospective study of nonoperative management. J Trauma. 1994;37(5):737-44.

14. von Bahten LC, Smaniotto $B$, Kondo $W$, Vasconcelos CN, Range M, Laux GL. Papel da laparoscopia no trauma abdominal penetrante. Rev Col Bras Cir. 2005;32(3):127-31

15. Friese RS, Coln CE, Gentilello LM. Laparoscopy is sufficient to exclude occult diaphragm injury after penetrating abdominal trauma. Trauma. 2003;58(4):789-92.

16. Waldschmidt ML, Laws HL. Injuries of the diaphragm. J Trauma. 1980;20(7):587-92.

17. Leppäniemi A, Haapiainen R. Occult diaphragmatic injuries caused by stab wounds. J Trauma. 2003;55(4):646-50.

18. Chen JC, Wilson SE. Diaphragmatic injuries: recognition and management in sixty-two patients. Am Surg. 1991;57(12):810-5

Recebido em 13/01/2011

Aceito para publicação em 18/03/2011

Conflito de interesse: nenhum

Fonte de financiamento: nenhuma

\section{Como citar este artigo:}

Saad Júnior R, Gonçalves R. Toda lesão do diafragma por ferimento penetrante deve ser suturada? Rev Col Bras Cir. [periódico na Internet] 2012; 39(3). Disponível em URL: http://www.scielo.br/rcbc

\section{Endereço para correspondência:}

Roberto Saad Júnior

E-mail: rsaad@uol.com.br 